\title{
PERFUSIÓN HIPOTÉRMICA DEL RIÑÓN AISLADO: RESPUESTA HIDRODINÁMICA Y BIOQUÍMICA AL PERFIL DE APLICACIÓN DE LA PRESIÓN
}

\author{
E. LLEDÓ GARCÍA, C. HERNÁNDEZ FERNÁNDEZ, J.I. MARTÍNEZ-SALAMANCA, \\ M. MORALEJO GÁRATE, R. CABELLO BENAVENTE, D. SUBIRÁ RÍOS, \\ J.F. DEL CAÑIZO LÓPEZ*
}

Servicio de Urología. *Depto. de Medicina y Cirugía Experimental. Hospital General Universitario Gregorio Marañón. Madrid.

Actas Urol Esp. 28 (7): 513-522, 2004

\section{RESUMEN}

PERFUSIÓN HIPOTÉRMICA DEL RIÑÓN AISLADO: RESPUESTA HIDRODINÁMICA Y BIOQUÍMICA AL PERFIL DE APLICACIÓN DE LA PRESIÓN

OBJETIVOS: Evaluar el efecto hidrodinámico, bioquímico e histológico de la presión positiva brusca en la perfusión del riñón aislado en hipotermia frente a la administración suave y progresiva de la solución de perfusión.

MATERIAL Y MÉTODOS: 16 perfusiones hipotérmicas de $180 \mathrm{~min}$. llevadas a cabo en condiciones de flujo constante en riñones de cerdo isogénico miniatura. Se utilizó una bomba de vacío regulada informáticamente. Se compararon los resultados obtenidos por los riñones prefundidos de forma brusca (I.B.) frente a aquellos prefundidos de forma suave y progresiva (I.S.).

RESULTADOS: A pesar de que ambos grupos de órganos consiguieron flujos de perfusión medios similares, el grupo I.B. mostró valores medios de presión de perfusión y resistencia vascular mayores. La concentración de metabolitos de óxido nítrico fue también más alta en el grupo I.B. Los riñones del grupo I.B. expusieron más edema tubular y retracción glomerular.

CONCLUSIONES: La perfusión hipotérmica brusca y forzada del riñón aislado no permite conseguir un flujo de perfusión mayor. Al contrario, de forma global los resultados hidrodinámicos e histológicos son de peor calidad en comparación a los órganos prefundidos de forma suave y progresiva.

PALABRAS CLAVE: Trasplante renal. Preservación renal. Perfusión.

\section{ABSTRACT \\ HYDRODYNAMIC AND BIOCHEMICAL ADAPTATION TO PERFUSION PRESSURE PROFILE IN ISOLATED HYPOTHERMIC RENAL PERFUSION}

OBJECTIVES: To evaluate hydrodynamic, biochemical and pathologic consequences of positive forced pressure on hypothermic isolated renal perfusions in comparison to softly progressive administration of perfusion solution.

MATERIAL AND METHOD: 16 hypothermic experimental pig renal perfusion o 180 min completed under constant flow conditions. A vacuum computer-controlled pump was used. A comparison was performed of the results obtained by two groups of organs (forced perfusion pressure -I.B.- and soft perfusion pressure -I.S.).

RESULTS: Even though perfusion flor did not offer significant differences among both groups at the end of the procedure, I.B. group showed medium perfusion pressure and renal vascular resistance values significantly higher. Nitric oxide metabolites concentration was also greater in I.B. group. Finally, these kidneys showed more tubular cell aedema and glomerular shrinkage in comparison to I.S. group.

CONCLUSIONS: Sharp and forced hypothermic perfusion of the isolated kidney don't increase flow perfusion values. Inversely, hydrodynamic and pathologic results are worst in comparison to soft and progressive organ perfusion.

KEY WORDS: Kidney transplant. Renal preservation. Perfusion. 


\section{OBJ ETIVOS}

Como objetivo principal analizamos el efecto hidrodinámico de la presión positiva forzada en la microcirculación renal. Para ello, hemos evaluado en perfusiones hipotérmicas $\left(4^{\circ} \mathrm{C}\right)$ de riñón aislado llevadas a cabo bajo condiciones de flujo constante $(0,85 \mathrm{ml} / \mathrm{min} / \mathrm{gr})$ la influencia del perfil de incremento inicial de la presión de perfusión (suave frente a brusco).

Utilizamos como parámetros de estudio la resistencia vascular renal (R.V.R.) y variación de la presión de perfusión media (P.P.R.).

Estudiamos además las consecuencias en el comportamiento bioquímico del riñón en perfusión, especialmente en relación a la neoformación de metabolitos de óxido nítrico (O.N.) -nitritos y nitratos- y liberación de LDH. Finalmente, evaluamos las alteraciones resultantes estructurales en el riñón (tubular y microvascular).

\section{MATERIAL Y MÉTODO}

1. El sistema de perfusión controlado por ordenador se describe en la Figura 1. Los riñones fueron perfundidos a flujo constante relacionado con el peso del órgano según una constante de conversión $(0,8 \mathrm{ml} / \mathrm{gr} / \mathrm{min})$, cuyo cálculo hemos descrito en publicaciones previas ${ }^{1}$. La presión de perfusión es controlada por un sistema informático pudiendo ser ésta dispensada de forma brusca o con incrementos suaves y progresivos de forma predeterminada. Cada 10 min. el ordenador registra las ondas instantáneas de flujo y presión durante un ciclo completo de la bomba.

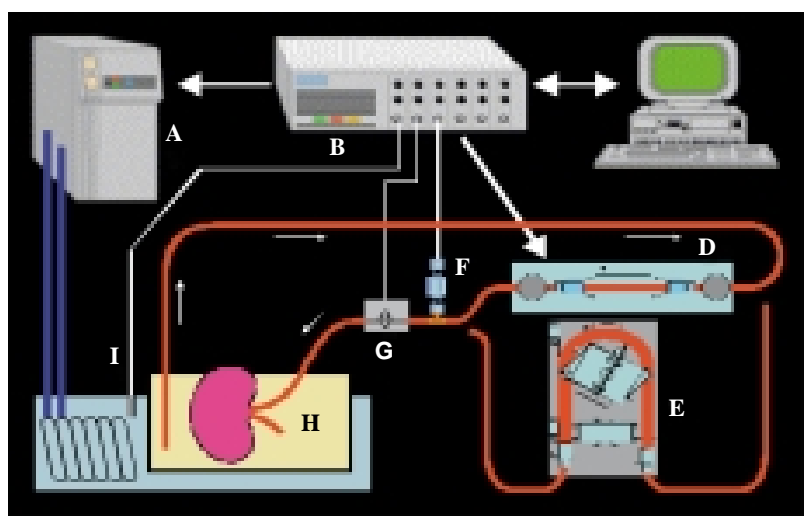

FIGURA 1. Sistema de perfusión renal extracorpóreo: A) Sistema de frio; B) Interface electrónica; C) Control; D) Bomba pulsátil; E) bomba rodillos; F) Transductor presión; G) Transductor flujo; H) Cámara órgano; I) Transductor temperatura.
Podemos evaluar de forma continua y en tiempo real a través del monitor la evolución del flujo vascular renal (F.V.R.), P.P.R. y R.V.R.

- Bomba pulsátil ${ }^{1,2}$. Es una verdadera bomba pulsátil desarrollada por nuestro grupo y controlada por una consola informatizada. El dispositivo de bombeo consiste en una cámara rígida externa (carcasa de metacrilato) y una cámara elástica interna fabricada en poliuretano elástico de grado biomédico (Fig. 2). En la cámara rígida se aplica presión negativa mediante una fuente de vacío controlada por la consola. Este vacío generado produce una expansión de la cámara elástica tubular. En un determinado momento, la consola detiene la aplicación de vacío y conecta la cámara rígida con la atmósfera. Esto induce la recuperación elástica de las paredes de la cámara y la impulsión de la solución de perfusión. Dos sistemas valvulares aplicados en los tubos de entrada y salida bajo el control de la consola aseguran la dirección del flujo (Fig. 3). Esta bomba permite obtener una

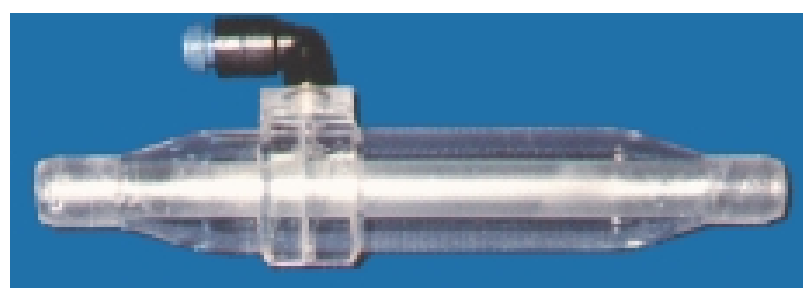

FIGURA 2. Bomba tubular gobernada por vacio.

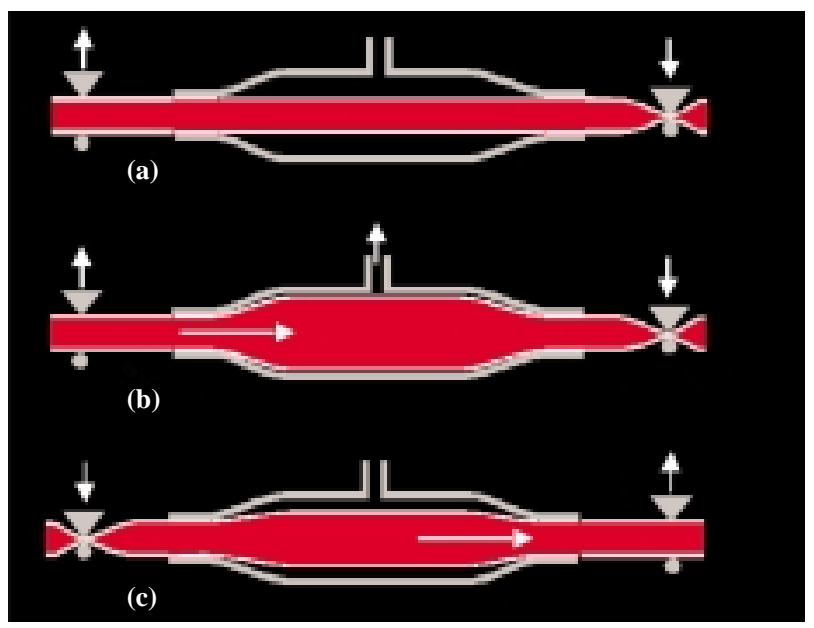

FIGURA 3. a) y b) Bomba de vacio-diástole: cierre válvula de salida, apertura válvula de entrada aplicación de vacio (expansión membrana); c) Bomba de vacio-sistole: apertura válvula de salida, cierre válvula de entrada, aplicación de presión atmosférica (retorno de membrana). 
onda de presión similar a la generada en el sistema circulatorio humano, con valores sistólico, diastólico y medio (Fig. 4).

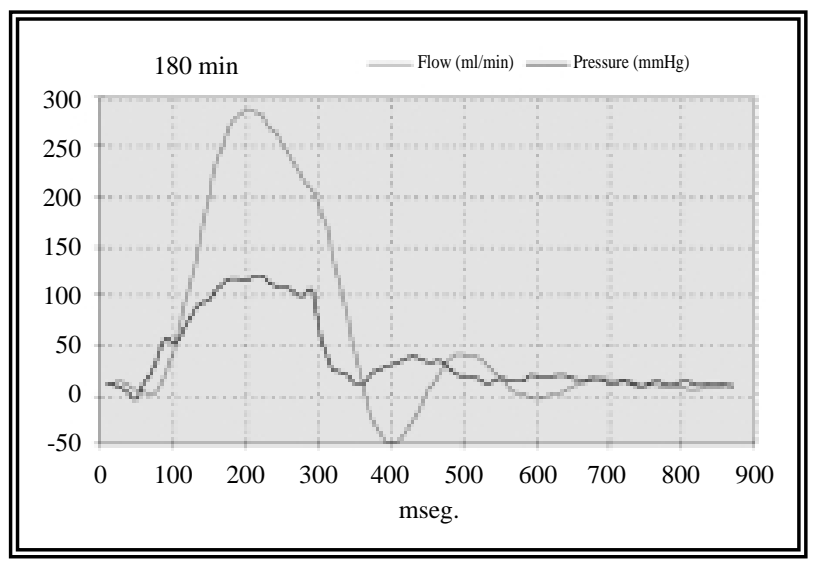

FIGURA 4. Ondas de presión instantánea (negro) y flujo (gris) en un riñón prefundido de inmediato tras su extracción en bomba de vacio durante 180 minutos.

- El sistema de enfriamiento utilizado, modos de medida de flujo, presión y resistencia vascular renal han sido descritos en publicaciones anteriores $^{1,2}$. El sistema de control estuvo basado en un ordenador personal equipado con una tarjeta de adquisición de datos (Keithley Metrabyte DAS1600). El software de control se desarrolló utilizando el programa Test Point (Capital Equipment Corp.). Finalmente, la interface electrónica fue diseñada también por nuestro grupo. Contiene todos los amplificadores de entrada y circuitos de salida requeridos para adaptar los niveles de señal a la tarjeta de adquisición de datos.

\section{Animales y procedimiento quirúrgico.} Utilizamos 8 cerdos isogénicos miniatura.

El manejo de los animales se ha realizado siguiendo la normativa legal según el Real Decreto 223/1988, de 14 de marzo (R. 601): animales. Protección de los utilizados para experimentación y otros fines científicos (Ministerio de Agricultura, Pesca y Alimentación), que incorpora las disposiciones legales, reglamentarias y administrativas de los estados miembros de la $\mathrm{CEE}$, respecto a la protección de los animales utilizados para experimentación y otros fines científicos, de la Directiva del Consejo 86/609/CEE (Leg. CC. EE. 4390) aprobada el 24 de noviembre de 1996.
3. Procedimiento de perfusión. Diseñamos un protocolo de trabajo sobre perfusiones llevadas a cabo a flujo constante con solución Belzer. Se prestableció un límite máximo de presión media de perfusión (100 mmHg). La perfusión se implementaba administrando un valor de flujo correspondiente al precalculado según peso, dispensando la perfusión de forma brusca y directa (Grupo I.B.) o bien de manera suave y progresiva (Grupo I.S.). El procedimiento experimental de manejo en el laboratorio ha sido descrito en publicaciones previas ${ }^{1,2}$. Se realizaron 16 perfusiones de riñón aislado cada riñón de la pareja a un grupo. Los riñones eran colocados en la cámara de órgano conectándose la cánula arterial al sistema de perfusión antes de iniciar el control de presión y la adquisición de datos. La perfusión era mantenida durante 180 min. A los 0, 60, 120 y $180 \mathrm{~min}$. se tomaban muestras de líquido de perfusión para su estudio bioquímico. Analizamos los distintos comportamientos hidrodinámicos de los riñones según fueran perfundidos inicialmente con presiones altas administradas bruscamente (inicio brusco: I.B.), frente a aquellos en los que la presión inicial de perfusión era baja y se incrementaba progresivamente (inicio suave: I.S.).

4. Método Estadístico. Las comparaciones de medias fueron realizadas mediante T-Student. Se estudió la asociación entre variables mediante un análisis de regresión. Se estableció el nivel de significación $\alpha$ en 0,05 .

\section{RESULTADOS}

En la Tabla 1 se presentan las diferencias en el análisis comparativo de las variables estudiadas para el factor incremento inicial de P.P.R. (inicio brusco frente a inicio suave: I.B.I.S.).

En esta tabla podemos observar no sólo la influencia de significación en las variables sino también la diferencia porcentual de los valores absolutos para I.S. frente a I.B.

Realizamos el análisis de la variación de los parámetros hidrodinámicos y bioquímicos durante la perfusión. Ello permite observar los incrementos absolutos y netos en relación al tiempo (Tablas 1 y 2). 
Tabla 1. Análisis comparativo global I.B.-I.S.

\begin{tabular}{lcccc}
\hline & Inicio & & & \\
& I.B. & I.S. & P & \\
\hline Inc. peso & 4,89 & 3,57 & 0,703 & $-27,0$ \\
Flujo & 71,47 & 69,78 & 0,432 & $-2,4$ \\
Presión & 58,00 & 43,50 & 0,000 & $-25,0$ \\
Resist. & 1,12 & 0,94 & 0,135 & $-16,1$ \\
Calcio (mmol/l) & 0,71 & 0,96 & 0,000 & 35,2 \\
CPK (U/l) & 26,34 & 21,59 & 0,007 & $-18,0$ \\
CREA (mgr/dl) & 0,69 & 0,81 & 0,000 & 17,4 \\
F_ALC (U/1) & 6,80 & 15,34 & 0,000 & 125,6 \\
GOT (U/1) & 11,00 & 9,56 & 0,349 & $-13,1$ \\
GPT (U/l) & 2,98 & 2,38 & 0,000 & $-20,1$ \\
LDH (U/l) & 80,94 & 72,77 & 0,051 & $-10,1$ \\
OX NITR ( $\mu M)$ & 12,88 & 10,95 & 0,011 & $-15,0$ \\
Potasio (mEq/l) & 69,04 & 49,55 & 0,000 & $-28,2$ \\
PROTEÍNA & 1,94 & 2,00 & 0,002 & 3,1 \\
Sodio (mEq/l) & 68,55 & 69,44 & 0,097 & 1,3 \\
\hline
\end{tabular}

\section{Cambios ponderales. Hidrodinámica}

La aplicación de un perfil inicial brusco de incremento de la P.P.R. produjo mayores incrementos de peso en comparación a los incrementos suaves (27\% mayor), aunque sin llegar a ser la diferencia significativa ( $\mathrm{p}=0,703)$. Los parámetros hidrodinámicos obtenidos mostraron P.P.R. media significativamente menor en los riñones con incremento inicial suave $(\mathrm{p}=0,000)$. El F.P.R. medio no ofreció diferencias significativas entre ambos grupos ( $p=0,432)$. La R.V.R. media de los órganos sometidos a incremento inicial suave de la P.P.R. fue menor durante todo el procedimiento, aunque sin llegar a la significación $(p=0,135)$. En cuanto a las variaciones netas, las diferencias entre los valores de P.P.R. media obtenidos fueron más importantes en los 5 primeros minutos para reducirse durante el resto de la perfusión. El F.P.R. medio fue mayor en el grupo I.B. en los $5 \mathrm{~min}$. iniciales, con valores posteriores superponibles. Sin embargo, la R.V.R. fue mayor en este grupo durante los 180 min. de perfusión (Figs. 5a y 5b).

Tabla 2. Variación dinámica absoluta y por intervalos de las variables durante la perfusión de 180 min. en relación al incremento inicial de presión de perfusión (I.B. frente a I.S.)

\begin{tabular}{|c|c|c|c|c|c|c|c|c|c|}
\hline \multicolumn{10}{|c|}{ Tiempo (minutos) } \\
\hline & & 5 & 60 & 120 & 180 & 5 & 60 & 120 & 180 \\
\hline \multirow[t]{2}{*}{ Flujo } & Brusco & 45,54 & 72,72 & 83,04 & 84,57 & & & & \\
\hline & Suave & 28,37 & 80,33 & 84,02 & 86,41 & & & & \\
\hline \multirow[t]{2}{*}{ Presión } & Brusco & 67,75 & 59,69 & 53,69 & 50,88 & & & & \\
\hline & Suave & 36,31 & 51,50 & 44,63 & 41,56 & 5 & 60 & 120 & 180 \\
\hline \multirow[t]{2}{*}{ Resist } & Brusco & 2,23 & 0,98 & 0,67 & 0,62 & 2,23 & 0,98 & 0,67 & 0,62 \\
\hline & Suave & 2,00 & 0,71 & 0,55 & 0,48 & 2,00 & 0,71 & 0,55 & 0,48 \\
\hline \multirow[t]{2}{*}{ Calcio } & Brusco & 0,64 & 0,69 & 0,76 & 0,74 & 0,64 & 0,05 & 0,07 & $-0,02$ \\
\hline & Suave & 0,86 & 0,92 & 1,03 & 1,04 & 0,86 & 0,06 & 0,11 & 0,01 \\
\hline \multirow[t]{2}{*}{ CPK } & Brusco & 9,69 & 25,63 & 33,13 & 36,94 & 9,69 & 15,94 & 7,5 & 3,81 \\
\hline & Suave & 6,13 & 21,00 & 27,50 & 31,75 & 6,13 & 14,87 & 6,5 & 4,25 \\
\hline \multirow[t]{2}{*}{ CREAT. } & Brusco & 0,66 & 0,68 & 0,72 & 0,71 & 0,66 & 0,02 & 0,04 & $-0,01$ \\
\hline & Suave & 0,78 & 0,81 & 0,82 & 0,83 & 0,78 & 0,03 & 0,01 & 0,01 \\
\hline \multirow[t]{2}{*}{ F_ALC } & Brusco & 2,51 & 7,94 & 8,38 & 8,38 & 2,51 & 5,43 & 0,44 & 0 \\
\hline & Suave & 2,50 & 16,31 & 19,00 & 23,56 & 2,5 & 13,81 & 2,69 & 4,56 \\
\hline \multirow[t]{2}{*}{ GOT } & Brusco & 5,81 & 12,50 & 13,13 & 12,56 & 5,81 & 6,69 & 0,63 & $-0,57$ \\
\hline & Suave & 1,69 & 10,31 & 12,88 & 13,38 & 1,69 & 8,62 & 2,57 & 0,5 \\
\hline \multirow[t]{2}{*}{ GPT } & Brusco & 2,00 & 2,81 & 3,50 & 3,63 & 2 & 0,81 & 0,69 & 0,13 \\
\hline & Suave & 1,38 & 2,44 & 3,00 & 2,69 & 1,38 & 1,06 & 0,56 & $-0,31$ \\
\hline \multirow[t]{2}{*}{ LDH } & Brusco & 37,50 & 80,69 & 98,00 & 107,56 & 37,5 & 43,19 & 17,31 & 9,56 \\
\hline & Suave & 24,38 & 70,50 & 90,19 & 106,00 & 24,38 & 46,12 & 19,69 & 15,81 \\
\hline \multirow[t]{2}{*}{ OXI NITRIC. } & Brusco & 9,83 & 12,66 & 14,64 & 14,63 & 9,83 & 3,09 & 1,98 & $-0,01$ \\
\hline & Suave & 9,57 & 10,37 & 11,72 & 11,88 & 9,57 & 0,54 & 1,35 & 0,16 \\
\hline \multirow[t]{2}{*}{ Potasio } & Brusco & 69,15 & 67,86 & 71,11 & 68,04 & 69,15 & $-1,29$ & 3,25 & $-3,07$ \\
\hline & Suave & 49,23 & 49,84 & 49,46 & 49,66 & 49,23 & 0,61 & $-0,38$ & 0,2 \\
\hline \multirow[t]{2}{*}{ PROTEÍNA } & Brusco & 1,97 & 1,93 & 1,93 & 1,93 & 1,97 & $-0,04$ & 0 & 0 \\
\hline & Suave & 2,01 & 2,01 & 2,01 & 1,99 & 2,01 & 0 & 0 & $-0,02$ \\
\hline \multirow[t]{2}{*}{ Sodio } & Brusco & 68,75 & 68,00 & 69,06 & 68,38 & 68,75 & $-0,75$ & 1,06 & $-0,68$ \\
\hline & Suave & 69,00 & 69,69 & 69,50 & 69,56 & 69 & 0,69 & $-0,19$ & 0,06 \\
\hline
\end{tabular}




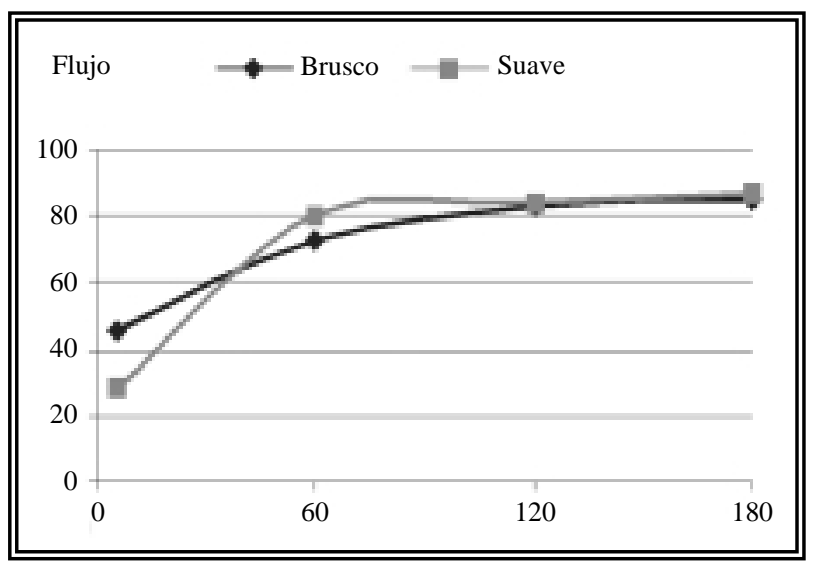

FIGURA 5a. Evolución comparativa de Flujo de Perfusión en relación al factor inicio: Inicio Brusco Vs Suave.

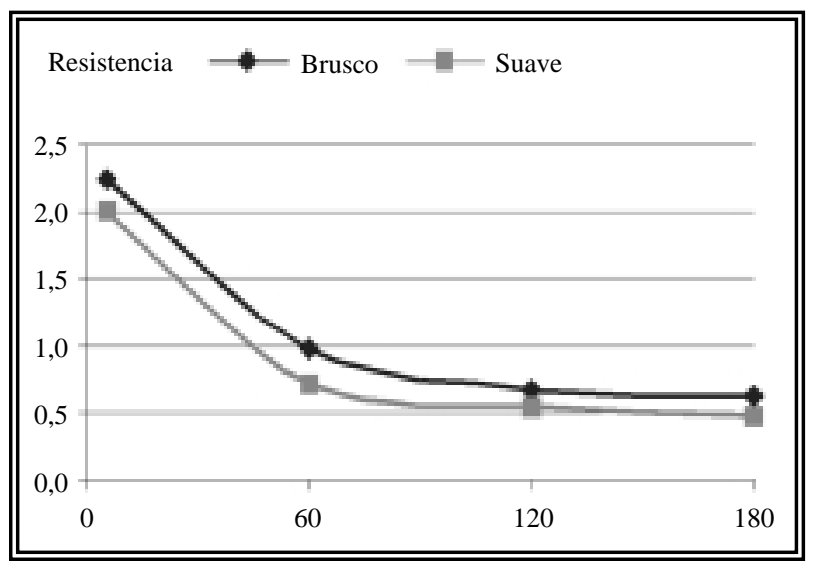

FIGURA 5b. Evolución comparativa de Resistencias Vasculares Renales en relación al factor inicio.

\section{Bioquímica}

En cuanto a los parámetros bioquímicos estudiados, pudimos observar:

- Calcio: la concentración media de calcio fue significativamente mayor $(\mathrm{p}=0,000)$ cuando se aplicó un incremento inicial suave de la P.P.R. El incremento neto de concentración fue especialmente importante en los 5 primeros minutos de perfusión, para reducirse las diferencias posteriormente.

- CPK: la concentración media de CPK fue significativamente mayor en los riñones sometidos a I.B. $(\mathrm{p}=0,007)$. Pudimos observar incrementos netos durante todo el proceso en ambos casos, aunque más marcados en los primeros 60 min. de perfusión.

- Fosfatasa alcalina: la concentración media de fosfatasa alcalina fue significativamente mayor en los incrementos iniciales suaves de
P.P.R. $(\mathrm{p}=0,000)$. El incremento neto fue sostenido durante todo el proceso y mayor en dicho grupo.

- GOT: no se observaron diferencias significativas en las concentraciones medias de GOT entre los grupos de riñones con I.B. o I.S. $(p=0,349)$. Los incrementos netos fueron más acusados en los primeros $60 \mathrm{~min}$. de perfusión en ambos casos.

- GPT: se pudo observar una concentración media significativamente mayor de GPT en los riñones con I.B. frente a I.S. $(\mathrm{p}=0,000)$. Los incrementos netos en la concentración de GPT fueron sostenidos y también mayores en los órganos con I.B.

- $L D H:$ aunque la concentración media de LDH en los órganos con I.B. fue mayor al compararla con la obtenida en el grupo I.S., la diferencia no fue significativa aunque sí mostró una clara tendencia $(\mathrm{p}=0,051)$. Los incrementos netos fueron mayores en el grupo I.S., salvo en la determinación a 5 min. (Figs. 6a y 6b).

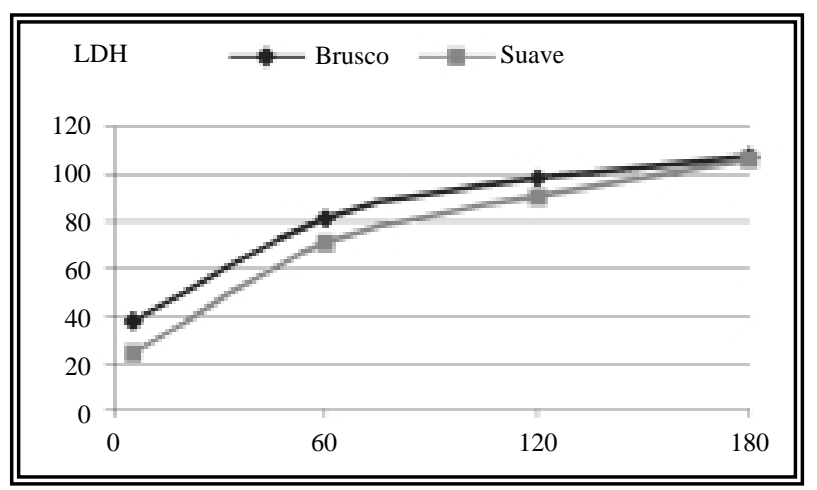

FIGURA 6a. Evolución de la concentración de LDH (U/I) durante la perfusión en relación al factor inicio.

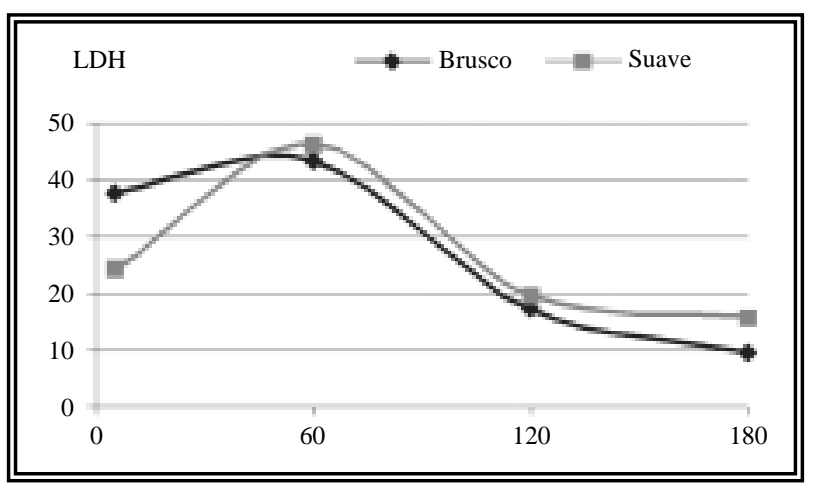

FIGURA 6b. Incrementos netos en la concentración de LDH (U/I) durante la perfusión en relación al factor inicio. 
- Nitritos y nitratos: la concentración media de nitritos y nitratos fue superior cuando se aplicó un incremento inicial brusco de P.P.R. en comparación a la obtenida con suave $(p=0,011)$. En el análisis evolutivo por tiempos, la concentración de nitritos y nitratos aumentó especialmente en los primeros 5 min. de perfusión en ambos casos, con valores absolutos mayores en el grupo I.B. frente a I.S. (Fig. 7a). Los incrementos netos fueron superponibles durante los $5 \mathrm{~min}$. iniciales de perfusión en ambos grupos. Posteriormente estos incrementos se redujeron aunque siguieron siendo algo mayores en los riñones con I.B. para superponerse a los 180 min. (Fig. 7b).

- Potasio y sodio: la concentración de potasio fue significativamente mayor en el grupo I.B. $(p=0,000)$. La concentración de sodio fue mayor en el grupo I.S., sin diferencia significativa aunque con tendencia a la significación ( $\mathrm{p}=0,097)$.

\section{Anatomía patológica}

En cuanto a los hallazgos anatomopatológicos, la aplicación de un perfil inicial brusco de incremento de presión de perfusión se relacionó con cambios más marcados de retracción glomerular y de edema tubular. Este hecho coincidía nuevamente con mayores aumentos de peso de los riñones perfundidos. La microcirculación renal permaneció indemne. Tampoco observamos un patrón característico de distribución zonal de las lesiones descritas.

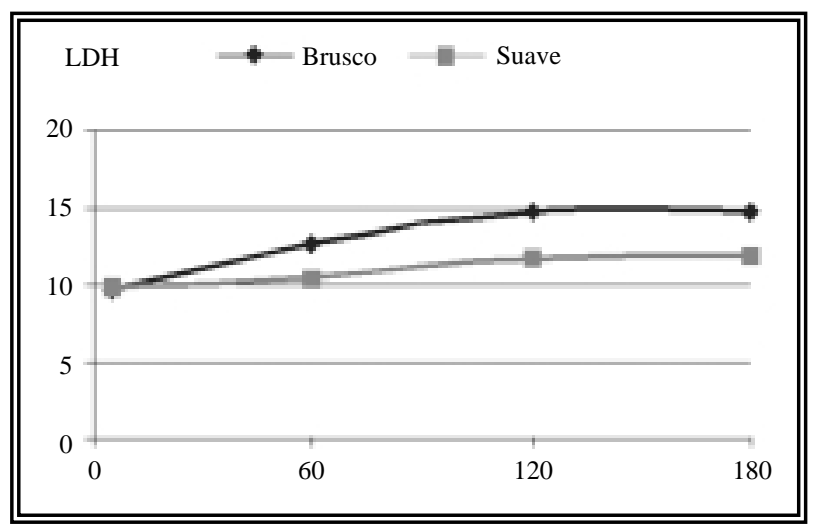

FIGURA 7a. Evolución de la concentración de Nitritos y Nitratos ( $\mu M)$ durante la perfusión en relación al factor inicio.

\section{DISCUSIÓN}

De la misma forma que la superioridad de pulsatilidad en la perfusión renal aislada hipotérmica no está totalmente demostrada, tampoco está claro el valor óptimo de la presión de perfusión ni las ventajas o inconvenientes de perfundir a presión o flujo constantes.

Grundmann publicó en 1975 un trabajo en el que se analizaban la presión de perfusión y flujo óptimos en un modelo de perfusión renal hipotérmica aislada ${ }^{3}$, utilizando diferentes valores de presión media. La valoración funcional de las perfusiones las realizaba analizando los flujos obtenidos durante la perfusión, así como el resultado funcional al trasplantar los órganos. Este autor obtenía los mejores resultados con presión media de $20 \mathrm{mmHg}$ en perfusión no pulsátil y de 20-30 mmHg con perfusión pulsátil. Estos valores de presión media se relacionaban con flujos medios de 0,8-1,3 ml/min/gr. Grundmann señalaba que presiones medias de perfusión inferiores a $20 \mathrm{mmHg}$ tenían consecuencias funcionales nefastas, lo que relacionaba con una imposibilidad de apertura de la microcirculación renal. Por otro lado, valores de presión media de perfusión superiores a $30 \mathrm{mmHg}$ en modelos pulsátiles se relacionan en este trabajo también con peores resultados funcionales. Ello podría ser consecuencia según el autor de edema secundario al trauma hidrodinámico celular, lo que podría producir obstrucción vascular compresiva durante el proceso limitando la calidad de la perfusión, así como una falta de revascularización efectiva tras el trasplante del órgano (fenómeno del non-reflow).

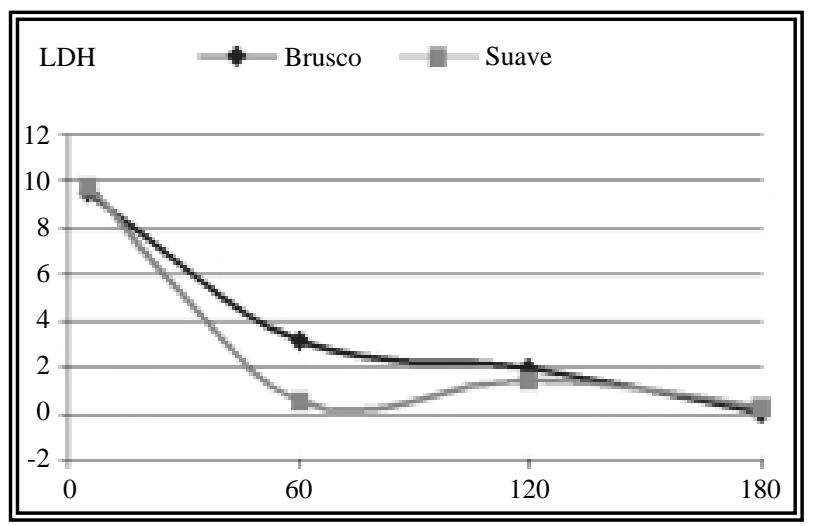

FIGURA $7 b$. Incrementos netos en la concentración de Nitritos y Nitratos $(\mu M)$ durante la perfusión en relación al factor inicio. 
Grundmann afirmaba que valores de presión de perfusión media de 20-30 mmHg, permitirian mantener mejor la integridad de la microvascularización renal al producir menos edema tisular.

Cerra $^{4}$ estudia el efecto de la pulsatilidad de la perfusión y de la presión oncótica de la solución utilizada en la aparición de lesiones vasculares endoteliales y subendoteliales, comparándolo con la acción de la hipotermia simple. Todos los órganos sometidos a perfusión pulsátil mostraron lesiones del endotelio vascular y de la membrana basal, con aparición de edema intersticial, tanto más importante cuanto mayor fuera la presión de perfusión y menor la presión oncótica del líquido. Cerra establece como valor crítico de presión de perfusión $60 \mathrm{mmHg}$. Este autor encuentra relación entre las lesiones endotelio-subendoteliales y la aparición de oclusiones vasculares trombóticas tras el trasplante, similares a las observadas en los procesos de rechazo inmunológico hiperagudo del injerto.

Los trabajos de Coyne ${ }^{5}$ están en consonancia con las conclusiones de Cerra ${ }^{4}$. Este autor observa que la utilización de presiones medias de perfusión renal hipotérmica superiores a $60 \mathrm{mmHg}$, se relacionan con fracaso funcional del órgano tras trasplante. Ellos relacionan este hecho con edema tisular, obstrucción tubular luminal y del flujo vascular renal efluente (fenómeno del non reflow).

Como ya se ha explicado anteriormente, la hidrodinámica del riñón perfundido en hipotermia es distinta a la hemodinámica del órgano en condiciones fisiológicas. En este último caso los procesos de autorregulación renal hacen que el flujo sanguíneo del órgano tienda a mantenerse constante. Por tanto, en normotermia la R.V.R. cambia con las variaciones de presión media de perfusión, como comprobaron McNay ${ }^{6}$. Benjamin ${ }^{7}$ demostraron en condiciones experimentales que en situación de hipotermia moderada $\left(20-25^{\circ} \mathrm{C}\right)$ el valor de resistencia vascular se encontraba en el límite inferior del rango de autorregulación; sin embargo, a $4^{\circ} \mathrm{C}$ el flujo puede variar de forma lineal con la presión media de perfusión ${ }^{3}$, pudiéndose establecer una relación entre valores de flujo para determinadas presiones de perfusión. Esto quiere decir que la disminución progresiva de la temperatura provoca una atenuación de los mecanismos de autorregulación renal.
Algunos autores restan importancia al hecho de predefinir un valor de presión de perfusión. Se puede ajustar un flujo determinado al inicio del procedimiento. De esta forma puede evitarse una perfusión renal deficiente si se establece un flujo mayor a $0,8 \mathrm{ml} / \mathrm{min} / \mathrm{gr}^{3}$, incluso tras la acción de ciertos factores isquémicos conocidos (isquemia normotérmica, etc.), en lugar de una determinada presión de perfusión.

En nuestra experiencia el incremento de peso de los riñones a los que se aplicó un perfil brusco de P.P. inicial fue un $27 \%$ mayor frente a aquellos en los que el perfil fue suave. Esta diferencia, no obstante, no llegó a ser significativa. Los órganos del grupo I.B. mostraron más edema tubuloglomerular en el examen anatomopatológico. Por otro lado, cuando analizamos detenidamente los resultados hidrodinámicos pudimos detectar hechos muy interesantes. La P.P.R. media del grupo I.B. fue un $25 \%$ mayor en comparación al grupo I.S. (58 mmHg Vs 43 mmHg, p 0,000). Esta diferencia -estadísticamente significativa- se mantuvo durante todo el procedimiento. La R.V.R. de los riñones I.B. fue mayor, aunque no significativamente, al grupo I.S. Esto ocurrió gracias a que los valores de flujo obtenidos en ambos grupos de órganos fueron similares. Es decir, que el grupo I.B. no mostró flujos mayores -lo que hubiera podido significar un efecto beneficioso del perfil brusco de aplicación de la presión de perfusión-. Al contrario, estos riñones dieron muestras de peor calidad de perfusión (P.P.R. y R.V.R. mayores), lo que probablemente traduce peores consecuencias de esta forma de aplicar la presión de perfusión en la microcirculación del órgano.

Al estudiar la evolución temporal de estos factores durante el proceso de perfusión (Tabla 2), observamos que aunque el flujo de perfusión a 5 min. en los órganos I.S. fue inferior $(28,37$ $\mathrm{ml} / \mathrm{min}$.) a I.B. (45,54 ml/min.), el obtenido a $180 \mathrm{~min}$. fue superponible en ambos grupos (I.B.: $84,57 \mathrm{ml} / \mathrm{min}$. - I.S.: $86,41 \mathrm{ml} / \mathrm{min}$.). La presión de perfusión a 5 min. prácticamente duplicó en el caso de los riñones con I.B. al valor obtenido por I.S. $(67,75 \mathrm{mmHg}$ frente a $36,31 \mathrm{mmHg})$. En el grupo I.S. los valores de presión de perfusión se mostraron relativamente constantes. En el grupo I.B. la presión de perfusión descendió progresivamente desde el valor máximo obtenido a 5 min., 
manteniéndose siempre superior a los valores correspondientes en el grupo I.S. Los valores de R.V.R. fueron siempre inferiores en I.S. en comparación a I.B. (Fig. 4).

Los riñones I.S. experimentaron una mejor adaptación hidrodinámica que los riñones I.B.: el flujo de perfusión sólo fue mayor en I.B. a 5 min., a costa de un valor de presión de perfusión y una R.V.R. mayores. La observación global del procedimiento permite extraer dos ideas: la respuesta del lecho vascular renal a valores elevados de presión media de perfusión dispensadas bruscamente en comparación a perfiles suaves es netamente constrictora. Por otro lado, pese a que se consiga tanto en I.S. como en I.B. el flujo constante deseado, el valor de R.V.R. se mantiene superior en los riñones I.B. Parece que presiones elevadas y de acción brusca al inicio de la perfusión influyen negativamente en la respuesta microvascular durante todo el procedimiento restante, a pesar de que los flujos obtenidos sean similares. De nuevo esta observación nos hace pensar en una respuesta dinámica del lecho vascular renal perfundido.

En los Programas Clínicos de Trasplante de órganos sólidos, existen varias situaciones en las que frecuentemente se están aplicando presiones positivas forzadas, con el fin de acelerar y "mejorar" la perfusión. En primer lugar, en la perfusión transaórtica del cadáver se suelen aplicar manguitos de presión a la bolsa de solución, independientemente del estado de las arterias o de la edad/características del donante. En el banco, tras la cirugía de preparación del riñón se lleva a cabo un "lavado" previo al almacenamiento. No es raro que, en esta circunstancia se utilice también un manguito si no se observa que la solución circula de forma fluida a través de la arteria renal. En estas dos situaciones referidas parecería potencialmente menos lesivo para la microcirculación renal la utilización de perfusión por gravedad (a $100 \mathrm{~cm}$ de altura), esto es, por gradiente de presión y no con aplicación de presiones positivas forzadas. Finalmente en los grupos que utilizan la perfusión en máquina, es poco frecuente que se regule el perfil de aplicación de una determinada presión de perfusión. La isquemia caliente del cadáver en parada puede actuar de forma sinérgica a la agresión hidrodinámica hipotérmica, debido al peor estado del endotelio vascular de esos riñones.
Estos hechos han sido ya probados en la preservación de otros órganos. La aplicación de P.P.F. en las maniobras de lavado transarterial con soluciones de preservación de pulmones previamente a su implante, puede inducir cuadros de hipertensión pulmonar en el post-operatorio inmediato, en comparación a la perfusión por gravedad $^{8}$.

En cuanto a la bioquímica vamos a diferenciar en nuestro trabajo dos grupos de moléculas según su evolución en la solución de perfusión:

1. Sustancias que mostraron mayor concentración en los órganos que habían sido perfundidos con incremento inicial de presión brusco: $\mathrm{CPK}$, GOT, GPT, LDH, metabolitos del O.N. y potasio. Debemos distinguir las moléculas con diferencias significativas frente a aquellas que no mostraron significación. Por un lado observamos la evolución de CPK, GPT, metabolitos del O.N. y potasio. Estas sustancias mostraron una concentración significativamente mayor en la solución de perfusión de los riñones I.B., en comparación a I.S. CPK y GPT que aumentaron progresivamente su concentración en la solución durante todo el proceso. Sin embargo, la concentración media comparativa I.B.-I.S. de potasio $(69,04 \mathrm{mEq} / 1$ frente a $49,55 \mathrm{mEq} / 1, \mathrm{p}<0,001)$ reflejó esencialmente la evolución durante los primeros 5 min. $(69,15$ $\mathrm{mEq} / \mathrm{l}$ frente a $49,23 \mathrm{mEq} / \mathrm{l})$. En las determinaciones a 60,120 y $180 \mathrm{~min}$. las diferencias entre ambos modelos (I.B.-I.S.) se mantuvieron prácticamente constantes, con concentraciones a 180 min., superponibles a las conseguidas a $5 \mathrm{~min}$. $(68,04 \mathrm{mEq} / 1$ frente a $49,66 \mathrm{mEq} / \mathrm{l})$. Entre modelos iguales de perfusión renal donde la única diferencia es el perfil de incremento inicial de presión, la aplicación de perfiles bruscos produce un efecto de "lavado" sobre el potasio detectado durante los $5 \mathrm{~min}$. iniciales del proceso.

El perfil evolutivo de los metabolitos del O.N. (Fig. 7) en relación a los parámetros hidrodinámicos en las condiciones evaluadas ofreció peculiaridades. No se detectó presencia de metabolitos del O.N. en la solución de los grupos I.B. e I.S. antes de iniciar la perfusión. En la comparación I.B.-I.S. los metabolitos del O.N. no mostraron concentraciones mayores en los riñones con valores de resistencia vascular menor. En el grupo 
I.B., a la vez que pudimos observar una concentración media de metabolitos del O.N. significativamente más alta que la del grupo I.S. (p 0,01), obtuvimos una P.P.R. media significativamente mayor (p 0,00) y una R.V.R. media también más alta, aunque, como ya se ha referido, sin llegar a ser la diferencia significativa (p 0,135). Por tanto, parece que la mayor distensión de la pared vascular por incrementos bruscos de la presión de perfusión produce incrementos más altos de metabolitos del O.N. en la solución.

Nuestro grupo ha demostrado en trabajos previos la neoformación de metabolitos de O.N. en perfusiones hipodérmicas de riñón aislado, así como la relación inversa entre los valores de R.V.R. y las concentraciones de dichos metabolitos ${ }^{9}$. Sin embargo, el hallazgo del presente experimento puede traducir un estímulo favorecedor alternativo de la síntesis de O.N. por el endotelio vascular: el shear-stress (estímulo bioquímico-mecánico de producción de O.N. por distensión brusca de la luz vascular) $^{10,11}$. El shear-stress puede generar producción de O.N. por el endotelio vascular mediante dos mecanismos: aumento del flujo de perfusión o vasoconstricción con flujo mantenido ${ }^{11}$. $\mathrm{El}$ incremento del flujo de líquido puede actuar sobre el endotelio mediante vías bioquímicas alternativas para producir O.N., según sea brusco y puntual o mantenido en el tiempo ${ }^{12}$. Resulta interesante observar en nuestro trabajo que las concentraciones de metabolitos del O.N. no tendieron a igualarse entre los grupos I.B. e I.S. durante la perfusión. El efecto del incremento inicial brusco de presión pareció sostenido durante todo el proceso, puesto que los valores de metabolitos del O.N. fueron más altos en el grupo I.B. en todas las determinaciones. A pesar de ello, y aunque sin llegar a la significación estadística, la R.V.R. fue mayor en el grupo I.B., por lo que puede existir algún otro tipo de respuesta dinámica vascular contráctil simultánea a la teórica producción de O.N. y también desencadenada por el perfil de incremento de presión, puesto que no observamos obstrucciones vasculares trombóticas ni mayor destrucción celular endotelial en el estudio patológico posterior. Algunos trabajos han mostrado evidencia de la producción de endotelina-1 (ET-1) por el endotelio vascular en respuesta al shear-stress, lo que podría ser inhibido por el GMPc ${ }^{13}$.
LDH mostró diferencias casi significativas en la comparación I.B.-I.S. $(80,94 \mathrm{U} / 1$ frente a $72,77 \mathrm{U} / 1$, p 0,051). Sin embargo, los incrementos netos por tiempos (60, 120 y $180 \mathrm{~min}$.) fueron mayores en el grupo I.S., coincidiendo con cifras de R.V.R. menores y F.P.R. mayores comparativamente frente a I.B. Sólo el incremento neto de LDH a 5 min. fue superior en I.B. (momento en el que el F.P.R. fue también mayor en I.B.).

2. Sustancias que mostraron mayor concentración en los órganos que habían sido perfundidos con incremento inicial de presión suave: calcio, creatinina, fosfatasa alcalina y sodio mostraron este comportamiento. Únicamente el sodio no mostró diferencia significativa en la comparación I.S.-I.B. ( $p>0,05)$. Calcio, creatinina y fosfatasa alcalina mostraron incrementos progresivos durante la perfusión con diferencias significativas ( $p<0,001$ en los tres casos). Este incremento fue más importante en el caso de la fosfatasa alcalina. En esta molécula observamos un comportamiento peculiar. La concentración comparativa I.S.-I.B. a $5 \mathrm{~min}$. fue casi idéntica $(2,5 \mathrm{U} / 1$ frente a 2,51 U/1). Sin embargo, las diferencias fueron incrementándose a lo largo de la perfusión; parecía que era el efecto sostenido de presiones de perfusión comparativamente mayores más que el perfil de incremento inicial lo que inducía su liberación.

\section{CONCLUSIONES}

La aplicación brusca de presiones de perfusión al riñón aislado en hipotermia $\left(4^{\circ} \mathrm{C}\right)$ produce una respuesta hidrodinámica de peor calidad en comparación a la perfusión suave y progresiva. Aunque el flujo vascular medio final de los riñones sometidos a perfusión fue similar independientemente del perfil de aplicación de la presión, ésto se consiguió a costa de una presión media y de unas resistencias vasculares mayores. Al analizar la producción de metabolitos del O.N. según el factor tipo de inicio la aplicación de altas presiones de perfusión de forma brusca se acompañó de mayores concentraciones de metabolitos del O.N. en el medio, aunque al mismo tiempo de valores más altos de R.V.R. 


\section{REFERENCIAS}

1. LLEDÓ GARCÍA E, HERNÁNDEZ FERNÁNDEZ C, LLORENTE ABARCA C, DEL CAÑIZO JF.: Sistema de perfusión hipotérmica de riñón aislado controlado por ordenador. Actas Urol Esp 2002; 26 (2): 77 84.

2. LLEDÓ GARCÍA E, HERNÁNDEZ C, DÍEZCORDERO JM, GARCÍA-BARRENO P, DEL CAÑIZO LÓPEZ JF.: Hydrodynamic and biochemical effects of isolated hypothermic renal perfusion depending on the pump model and perfusion solution. Transpl Proc 2003; 35: 1661-1663.

3. GRUNDMANN R, RAAB M, MEUSEL E, KIRCHHOFF R, PICHLMAIER H.: Analysis of the optimal perfusion pressure and flow rate of the renal vascular resistance and oxygen consumption in the hypothermic perfused kidney. Surgery 1975; 77 (3): 451-461.

4. CERRA FB, RAZA S, ANDRES GA, SIEGEL JH.: The endotelial damage of pulsatile renal preservation and its relationship to perfusion pressure and colloid osmotic pressure. Surgery 1977; 81 (5): 534541.

5. COYNE JA, RUDOLF LE, ALLPORT EL. Perfusion pressure and its relation to vascular resistance in isolated canine kidneys. In "Organ Perfusion and Preservation” (JC Norman ed). Appleton, New York, 1968: 33-34.

6. McNAY JL, ABE Y.: Redistribution of cortical blood flowduring renal vasodilatation in dogs. Circ Res 1970; 27 (6): 1023-1032.

7. BENJAMIN JL, SELL KW.: Effects of temperature on kidneys preserved by hypothermic perfusion. Transplantation 1972; 14 (4): 501-510.

8. DeCAMPOS K, KESHAVJE S, SLUTSKY AS, MINGYAO L.: Alveolar recruitment prevents rapid reperfusion-induced injury of lung transplants. The Journal of Heart and Lung Transplantation 1999; 18 (11): 1096-1102.

9. LLEDÓ GARCÍA E, SUBIRÁ D, CASTAÑO I, MORALEJO M, MARTÍNEZ SALAMANCA J, CABELLO R, DEL CAÑIZO LÓPEZ JF, HERNÁNDEZ FERNÁNDEZ C.: Renal preservation by machine perfusion. Experimental computerized design. European Urology Supplements 2003 february; 2, Issue 1: 10

10. BUGA GM, GOLD ME, FUKUTO JM, IGNARRO LJ.: Shear-stress induced release of nitric oxide from endotelial cell growths on beads. Hypertension 1991; 17 (2): 187-193.

11. HECKER M, MÜLSCH A, BASSENGE E, BUSSE R.: Vasoconstriction and increased flow: two principal mechanisms of shear stress-dependent autacoid release. Am J Physiol 1993; 265:3 Pt 2, H828-33.

12. McALLISTER TN, FRANCOS JA.: Steady and transient fluid shear-stress stimulate NO release in osteoblasts through distinct biochemical pathways. $J$ Bone Min Res 1999; 14 (6): 930-936.

13. KUCHAN MJ, FRANGOS JA.: Shear stress regulates endothelin-1 release via rotein kinase $\mathrm{C}$ and cGMP in cultured endothelial cells. Am J Physiol 1993; 264:1 Pt2, H150-6.

Dr. E. Lledó García

(Servicio de Urología)

Hospital Gral Univ. Gregorio Marañón

C/ Doctor Esquerdo, 46

28007 Madrid

(Trabajo recibido el 15 diciembre de 2003) 